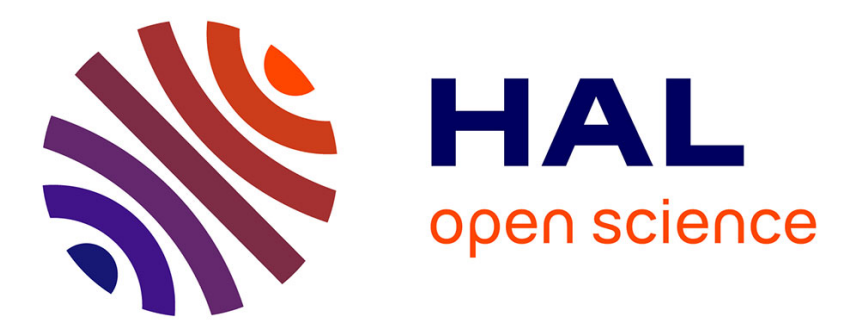

\title{
ATLAS electromagnetic barrel calorimeter performance in recent testbeams
}

R. Lafaye

\section{To cite this version:}

R. Lafaye. ATLAS electromagnetic barrel calorimeter performance in recent testbeams. X Pisa Meeting on Advanced Detectors: Frontier Detectors for Frontier Physics, May 2006, La Biodola, Italy. pp.82-84, 10.1016/j.nima.2006.10.350 . in2p3-00133492

\section{HAL Id: in2p3-00133492 https://hal.in2p3.fr/in2p3-00133492}

Submitted on 26 Feb 2007

HAL is a multi-disciplinary open access archive for the deposit and dissemination of scientific research documents, whether they are published or not. The documents may come from teaching and research institutions in France or abroad, or from public or private research centers.
L'archive ouverte pluridisciplinaire HAL, est destinée au dépôt et à la diffusion de documents scientifiques de niveau recherche, publiés ou non, émanant des établissements d'enseignement et de recherche français ou étrangers, des laboratoires publics ou privés. 


\title{
ATLAS Electromagnetic Barrel Calorimeter Performance in Recent Testbeams
}

\author{
R. Lafaye. \\ LAPP, Annecy, France \\ On behalf of the ATLAS LAr Collaboration
}

\begin{abstract}
During the combined test beam in summer 2004 a slice of the ATLAS barrel detector - including all detector sub systems from the inner tracker, the calorimetry to the muon system - was exposed to particle beams (electrons, pions, photons, muons) with different energies ( $1 \mathrm{GeV}$ to $350 \mathrm{GeV}$ ). The aim was to study the combined performance of the different detector sub systems in ATLAS-like conditions. We will present studies with different amounts of material in front of the calorimeter, representing various regions in the ATLAS detector, performance results from the electromagnetic calorimetry, including uniformity, resolution, and linearity as well as a discussion of the calibration scheme. The performance in the combined ATLASlike set-up will be compared to the one obtained in stand-alone testbeams.
\end{abstract}

Key words: , ATLAS, combined, testbeam, LAr, barrel PACS:

\section{Introduction}

For a Higgs boson decaying to two photons or to four electrons, in the mass range from 90 to $180 \mathrm{GeV}$, ATLAS should measure the Higgs mass with $1 \%$ precision using the calorimeter system alone. This translates into the requirements of a sampling term of less than $10 \% / \sqrt{E}$, associated with a global constant term of $0.7 \%$ and an angular resolution bet-

Email address:

Remi.Lafaye@cern.ch (R. Lafaye.). ter than $50 \mathrm{mrad} / \sqrt{(} E)$.

The technology chosen for the ATLAS electromagnetic calorimeter is lead and liquid argon sampling with accordion shaped electrodes and absorbers. Liquid argon calorimetry has been chosen because of its intrinsic linear behavior, stability of the response over time and radiation tolerance. The accordion geometry has been chosen because it allows a very high granularity and a very good hermiticity, since the high voltage and signal cables run only at the front and back faces of the detector. 
In addition, the accordion geometry minimizes inductances in the signal paths, allowing the use of the fast shaping needed for operation with 25 ns bunch intervals between collisions at LHC.

\section{The Testbeams}

A detailed description of the ATLAS barrel electromagnetic calorimeter can be found in ref[?]. Once the calorimeter is assembled, there is no discontinuity along the azimuthal angle $\phi$, but for ease of construction, a half barrel is divided into 16 modules. Four of these modules were tested in standalone testbeams during the years 2001 and 2002. Then, for the 2004 testbeam, combining a full slice of the ATLAS barrel detectors, an additional module was constructed.

During the combined testbeam, the module was read by the final electronics and operated by ATLAS TDAQ software. Electrons, pions, muons protons and photons with energies ranging between 1 to 350 $\mathrm{GeV}$ were collected. The pixels and SCT detectors of the tracker were installed in a magnet which was turned ON for part of the data taking.

\section{Energy Reconstruction}

The signal amplitude in each cell of the calorimeter is reconstructed using optimal filtering coefficients. The signal is converted to current using electrical calibration results and then to $\mathrm{GeV}$ using comparison with data and simulation. Corrections for Argon temperature and cross-talk are then applied to obtain the final energy measured in each cell. A complete description of the electronic calibration procedure and cell energy reconstruction can be found in ref[?,?].

Mosgt of the energy of an electromagnetic interacting particle is absorbed in the instrumented regions of the calorimeter. A small fraction is deposited in non-instrumented materials like the beam counters, the cryostat, the LAr and cables between the PS and the first accordion layer, the back of the calorimeter. Cells from the four layers are combined to form a cluster of $0.75 \times 0.75$ in the eta and phi using the following formula:

$$
\begin{aligned}
E_{r e c}= & a+b E_{P S}+c \sqrt{E_{P S} E_{1}} \\
& +d \sum_{i=1,3} E_{i}+e E_{3}
\end{aligned}
$$

$E_{P S}$ and $E_{i=1,3}$ are the cluster energies in the presampler and the three layers of the calorimeter. The calibration constants $a$ to $e$ can be derived from a Monte Carlo simulation. They depend sightly on the beam energy and on the upstream material.

The presampler is used to correct for the energy lost in the material upstream of the calorimeter, using the $a+b E_{P S}$ term. As demonstrated from simulation and data comparison, the energy lost in cables and support structure in between the presampler and the calorimeter can be estimated with the term $c \sqrt{E_{P S} E_{1}}$. Similarly, the energy deposited in the 
back layer can be related to the one leaking at the back of the calorimeter (term $e E_{3}$ ).

The cluster energy is then corrected for $(\eta, \phi)$ modulations, loss of signal in the electrode transition region, early lost bremsstrahlung and nuclear binding compensation between $\eta<0.8$ and $\eta>0.8$, were the lead thickness is different.

\section{Energy Linearity, Resolution and Uniformity}

During 2002 stand-alone testbeams, the beam energy was under control with an uncertainty of $3 \times 10^{-4}$. This precision was reached thanks to a magnetic spectrometer, which bending magnets were equipped with a precision power supply, together with a sophisticated calibration of their bending power.

Using the energy reconstruction scheme summarized above a linearity of the energy response of $0.1 \%$ has been reached for the energy range from 15 to $180 \mathrm{GeV}$, as shown on figure ??. The fit on the resolution yields a sampling term of $10 \% / \sqrt{E(G e V)}$, a noise term of $200 \mathrm{MeV}$ and a local constant term of $0.2 \%$.

The corresponding uniformity (see figure ??) achieved on a region of $1.4 \times 0.2$ in $(\eta, \phi)$ on two of the tested production modules was $0.44 \%$ for a $250 \mathrm{GeV}$ beam with an energy resolution between 0.7 and $0.9 \%$.

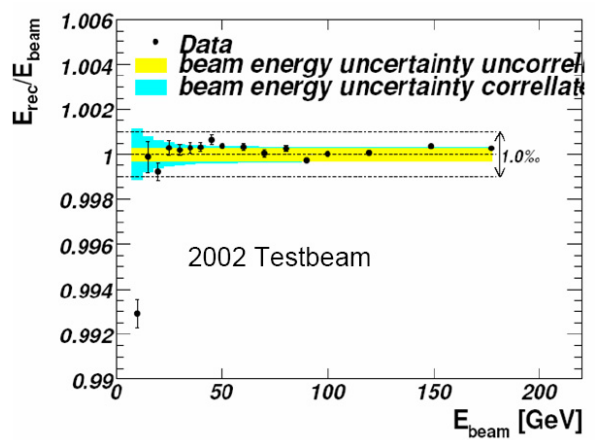

Fig. 1. Linearity obtained from 2002 beam test data, normalized to the 100 $\mathrm{GeV}$ point.

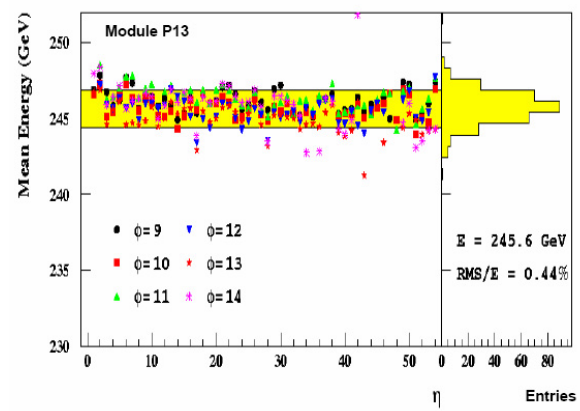

Fig. 2. Uniformity of the energy response as a function of $\eta$, obtained from 2002 beam test data for module P13.

\section{Material Studies}

To extend the results obtained in 2002 a dedicated material scan was carried out during the 2004 combined run. Different amounts of aluminum plates, placed in front of the outer cryostat wall, allowed to study the impact of close upstream material on energy reconstruction. New calibration constants were derived from Monte-Carlo simulation for all beam energies and upstream material configurations.

The linearity changed from $0.68 \%$ without additional upstream material to $1.4 \%$ with $75 \mathrm{~mm}$ of $\mathrm{Alu}-$ minium. Although, the beam energy 
was only precise to $5 \times 10^{-3}$ and thus dominates the linearity measurements. The resolution obtained shows very small variations, with a sampling term between 11.0 and $11.4 \% / \sqrt{E(G e V)}$ and a local constant term between 0.55 and $0.56 \%$.

\section{Combined studies}

Work is presently ongoing to complete analysis combining the ATLAS tracker and the electromagnetic calorimeter:

- comparing the impact point on the calorimeter measured from a reconstructed charged track and the reconstructed electron shower position has allowed to align the two detectors.

- Photons data were collected to allow the precise determination of the photon energy scale and to study the reconstruction of converted photons, in order to better constraint the simulation for the preparation of the Higgs analysis. Work is ongoing.

\section{Response to Muons}

The calorimeter response to muon was shown to have a signal over noise ratio around 6.5 using optimal filtering coefficients method, with a noise of $\sigma=42.2 \pm 0.6 \mathrm{MeV}$ and muon signal of $\sigma=284.1 \pm 1.1 \mathrm{MeV}$.

\section{Conclusion and Acknowledg- ment}

The 2004 combined testbeam was a successful test of a full slice of the ATLAS detector. Many promising combined analyses are still ongoing, such as converted photons studies with the tracker and combined analysis with the hadronic calorimeter.

The author wants to thank the whole LAr Collaboration, and especially the combined testbeam team, for their support and contagious enthusiasm.

\section{References}

[1] B. Aubert et al., Construction, assembly and tests of the ATLAS electromagnetic barrel calorimeter, Nucl. Instrum. Meth. A558:388418,2006 .

[2] M. Aleksa et al., 2004 ATLAS Combined Testbeam: Computation and Validation of the Electronic Calibration

Constants for the Electromagnetic Calorimeter, ATLAS Note ATLLARG-PUB-2006-003.

[3] W. Lampl, Optimizing the Energy Measurement of the ATLAS Electromagnetic Calorimeter, CERN-THESIS-2006-002 